\title{
Formation of beam-plasma structures in pulsar magnetosphere
}

\author{
V.N. Mel'nik \\ Institute of Radio Astronomy, Krasnoznamyonnaya St.4, 310002, \\ Kharkov, Ukraine
}

\begin{abstract}
Formation of beam-plasma structure, a new non-linear object, in pulsar magnetosphere is considered.
\end{abstract}

In (Melrose 1992) Melrose formulated some difficulties in the theory of pulsar radioemission. One of them is the absence of bunching mechanism with velocity dispersion of particles. Recently a new nonlinear object, beam-plasma structure, analogous to soliton was found in the "electron beam+plasma" system (Mel'nik 1990; Mel'nik 1994; Mel'nik 1995; Mel'nik, Lapshin, \& Kontar 1999).

Let us consider formation of this object in pulsar magnetosphere. Suppose there is a monoenergetic electron beam of finite length (the boundary problem was considered in (Mel'nik 1994))

$$
f(v, x, t)=n_{b} \exp (-x / d) \delta\left(v-v_{0}\right) .
$$

In the case of the development of the kinetic stream instability in electronpositron plasma of pulsar magnetosphere, i.e., when $n_{b} / n \ll\left(\bar{\gamma} / \gamma_{b}\right)^{3}$, where $n_{b}\left(\gamma_{b}\right), n(\bar{\gamma})$ are the densities (the Lorentz factors) of beam and plasma (here we take the values in the plasma rest system). This condition can be realized in the scheme of Usov (Usov 1987), for example, for the parameters $n_{b} / n=10^{-4}$, $\bar{\gamma} / \gamma_{b}=10^{-1}$. Because of $n_{b} \gamma_{b} m c^{2} \ll n \bar{\gamma} m c^{2}$ the case of the weak turbulance should be analyzed. So for the electron distribution function $f(p, x, t)\left(n_{b}=\right.$ $\left.\int f(p) d p\right)$ and the spectral energy density of Langmuir waves $W(k, x, t)(W=$ $\int W(k) d k$ ) we have the system of kinetic equations

$$
\begin{gathered}
\frac{\partial f}{\partial t}+v \frac{\partial f}{\partial x}=\frac{4 \pi^{2} e^{2}}{c} \frac{\partial}{\partial p} W \frac{\partial f}{\partial p} \\
\frac{\partial W}{\partial t}+v_{g r} \frac{\partial W}{\partial x}=\frac{\sqrt{2} \pi}{4} \omega_{p e} \frac{m^{2} c^{2}}{n} \frac{\bar{\gamma}^{1 / 2}}{\gamma^{3}} \gamma^{3} \frac{\partial f}{\partial p} W, \quad \omega_{p e}=k v
\end{gathered}
$$

Here $\omega=c[k-\alpha(k-\bar{k})]\left(\alpha=1 / 12 \bar{\gamma}^{2}, \bar{k}=\sqrt{2 \bar{\gamma}} \omega_{p e} / c\right)$ is the dispersion law for Langmuir waves and $v_{g r}=\partial \omega / \partial k=c(1-\alpha)$ is their group velocity. Solving equations (2)-(3) we will be interested in electron flying-off time $t \gg \tau_{q}\left(\tau_{q}=\right.$ $\left(\omega_{p e} n_{b} \gamma_{b} / n \bar{\gamma}^{5 / 2}\right)^{-1}$ - quasilinear relaxation time). In this case we can turn from the kinetic description to gasdynamic one (Mel'nik 1990; Mel'nik 1995; Mel'nik, Lapshin, \& Kontar 1999). Then we suppose that at every point the plateau at the electron distribution function establishes in the momentum space

$$
f(p, x, t)=\left\{\begin{array}{l}
F(x, t), \quad p_{\min }<p<P(x, t) \\
0, \quad p>P(x, t) .
\end{array}\right.
$$


For the spectral energy density of Langmuir waves we have

$$
W(p, x, t)=\left\{\begin{array}{l}
W_{0}(p, x, t), \quad p_{\min }<p<P(x, t) \\
0, \quad p>P(x, t)
\end{array}\right.
$$

Integration of equations (2)-(3) with (4),(5) gives us the system of gasdynamic equations for $F(x, t), P(x, t), W_{0}(p, x, t)$

$$
\begin{gathered}
\frac{\partial F}{\partial t}+v_{p l} \frac{\partial F}{\partial x}=0, \quad \frac{\partial P}{\partial t}+\frac{P}{m \sqrt{1+P^{2} / m^{2} c^{2}}} \partial \frac{\partial P}{\partial x}=0, \\
\frac{\partial F}{\partial t}+v \frac{\partial F}{\partial x}=\frac{4}{\sqrt{2}} \frac{\overline{\gamma^{3}}}{\bar{\gamma}^{1 / 2}} \frac{\omega_{p e}}{m c^{3}} \frac{\partial}{\partial p} \frac{1}{\gamma^{3}}\left(\frac{\partial W_{0}}{\partial t}+v_{g r} \frac{\partial W_{0}}{\partial x}\right), \\
\left(\frac{\partial P}{\partial t}+v_{g r} \frac{\partial P}{\partial x}\right) W_{0}=0, \quad \frac{\partial W_{0}}{\partial t}+v_{g r} \frac{\partial W_{0}}{\partial x}=0, \quad p=P
\end{gathered}
$$

Solution of the gasdynamic equations (6)-(8) has the form

$$
\begin{gathered}
P(x, t)=\text { const }=p_{0}, \quad F(x, t)=\frac{n_{b}}{p_{0}-p_{\min }} \exp \left(-\frac{\left|x-v_{p l} t\right|}{d}\right) \\
W_{0}(p, x, t)=W_{b p s}(p, x, t)+W_{L w}(p, x, t)
\end{gathered}
$$

where $W_{b p s} \propto \partial F / \partial t$ and $W_{L w} \propto F\left(x-v_{g r} t\right)$.

The speed of beam-plasma structure is constant and is equal to $v_{p l}(\approx c)$ that has the Lorentz factor $\gamma_{b p s}=\sqrt{\gamma_{b}} \bar{\gamma}$. The profile of electron stream remains invariable. The energy of Langmuir waves that "accompany" electrons with the same speed is small in comparison with electron energy $\left(W_{b p s} / E \approx\right.$ $2 p_{\min } p_{0} / m^{2} c^{2} \gg 1$ ). But there is the cloud of Langmuir waves moving with the group velocity $v_{g r}$. Their energy is high $W_{L w} \approx E$. When $\gamma_{0}=6 \bar{\gamma}$ this cloud propagates together with beam-plasma structure. Because of there are two objects that consist of Langmuir waves they both can be sources of plasma emission. If the plasma radiation occurs with the help of fast electrons and Langmuir waves the most brightness temperature can be expected to be at the joint propagation of beam-plasma structure and Langmuir wave cloud.

At the propagation of electron beams with parameters, at which the stream kinetic instability is realized, a relativistic beam-plasma structure is formed in electron-positron plasma. The velocity dispersion in this structure is the cause of its formation. Thus one of the demands to bunching mechanism turnes out to be fulfilled for this structure. It can be hoped to allow to develop the theory of coherent curvature emission in application to pulsar radioemission.

\section{References}

Mel'nik, V.N. 1990 Ukrainian Fiz. Zhurn., 35, 871

Mel'nik, V.N. 1994 Plasma Phys.Rep., 20, 828

Mel'nik, V.N. 1995 Plasma Phys. Rep., 21, 94

Mel'nik, V.N. Lapshin, V. \& Kontar, E. 1999 Solar Phys., 184, 353

Melrose, D.B. 1992 in Proc.IAU Colloq.128, (Ziel. Gora: Pedag.Univ.Press) 306 Usov, V.V. 1987 ApJ, 320, 828 